\title{
Fatal Salmonella Typhi Necrotising Fasciitis following Intra-articular Steroid Injection
}

\author{
Sagar Narang, ${ }^{\mathrm{a}, \mathrm{c}}$ Prakash Sapkota ${ }^{\mathrm{b}, \mathrm{d}}$
}

\begin{abstract}
:
Intra-articular steroids administration in the absence of aseptic precautions can have disastrous consequences. Immunocompromised patients are at an increased risk of developing infections following such procedures. Salmonella has been infrequently reported as a causative organism for necrotising fasciitis. Gram negative endotoxemia with disseminated intravascular coagulation resulted in fatality in this patient. The case study is being presented to emphasise the need for aseptic precautions and sterile techniques while administering intra-articular steroids, to have a low threshold towards treating early joint infections expeditiously, and to consider possibility of a gram negative organism as a cause of septicaemia and necrotising fasciitis especially in debilitated patients.
\end{abstract}

Keywords: necrotising fasciitis • salmonella • steroid injection

\section{INTRODUCTION:}

Intra-articular steroid injections are routinely used by trained orthopaedic surgeons in the management of rheumatoid, gouty and osteoarthritis patients, painful conditions like adhesive capsulitis of the shoulder, plantar fasciitis, medial and lateral epicondylitis of the elbow. Minor complications like pain and inflammation at injection site are not uncommon following such injections. Rarely infection may occur, especially in immunocompromised patients.

Development of necrotising fasciitis (NF) following intra-articular steroid infiltration is extremely rare. Only five such cases have been reported previously in international literature. ${ }^{1-5}$ The bacterial isolates in these cases were either Streptococci or Staphylococci. Very few cases of Salmonella NF have been reported

\footnotetext{
a - Associate Professor

b - Lecturer

c - Department of Orthopedics and Traumatology

Lumbini Medical College Teaching Hospital, Palpa, Nepal

d - Department of Surgery

Lumbini Medical College Teaching Hospital, Palpa, Nepal
}

Corresponding Author:

Dr. Sagar Narang

e-mail: sagarnarang@jlmc.edu.np

How to cite this article:

Narang S, Sapkota P. Fatal salmonella typhi necrotising fasciitis following intra-articular steroid injection. Journal of Lumbini Medical College. 2013;1(2):125-7. doi:10.22502/jlmc.v1i2.36. in literature. ${ }^{6-11}$ This is probably the first reported case of Salmonella NF following intra-articular steroid injection.

Salmonella NF can be managed with antibiotics and supportive therapy when detected early. The patient presented here came to us moribund, in the stage of endotoxic shock when disseminated intravascular coagulation (DIC) had already set in. She later, succumbed to multi-system organ failure. Family members of the deceased were informed that the data concerning this case would be submitted for publication, and they consented.

\section{CASE REPORT:}

Local steroid injections are often administered by inadequately trained medical personnel, probably without due consideration being given to strict aseptic conditions. The presented case is an extreme form of an infective complication resulting in a fatality.

A 56-year-old lady presented to our emergency service in a toxic state. Her blood pressure was $90 / 60 \mathrm{~mm} \mathrm{Hg}$, she was febrile $\left(101^{\circ} \mathrm{F}\right)$ and delirious. Pallor, dehydration, tachycardia (120 beats/min) and tachypnea (respiratory rate 25/min) were present. Resuscitative measures were started and preliminary blood investigations sent. Examination of her left leg, showed open surgical incisions, consistent with those made for compartment release, located on the antero-lateral and posteromedial aspects. Both the incisions were infected, with necrotic subcutaneous tissue and fascia present throughout the length of the 
wound (Fig.1). The left leg felt firm to palpation, and induration involved the leg and extended to posteromedial aspect of proximal thigh. Bone-deep pressure sores were present over the sacrum $(5 \mathrm{~cm} \times 5 \mathrm{~cm})$ and both greater trochanters $(8 \mathrm{~cm} \times 5 \mathrm{~cm})$.

Two months earlier, the patient developed left knee pain, and attended a local hospital. She was diagnosed as osteoarthritis left knee and advised intra-articular steroid injection for her symptoms. The injection was administered by a health-care provider in his clinic. Two days later, the patient developed swelling and increasing pain on knee movement. Analgesics (aceclofenac $100 \mathrm{mg}$ twice a day) were used for five days without any improvement. The knee swelling increased and extended towards the left leg. The patient was taken back to the same clinic, where she was reassured that the swelling would gradually subside on rest, analgesics and limb elevation. This management was continued for next two weeks.

As the patient's general condition deteriorated, she was admitted to Hospital X. A diagnosis of post-injection cellulitis left leg was made. She was administered intravenous antibiotics (amoxicillin sodium + clavulanate potassium 1.2 gm thrice a day) for two weeks. No improvement resulted, and she developed fever. She was then transferred to Hospital $\mathrm{Y}$, where she was diagnosed as pyomyositis of calf muscles with compartment syndrome. Two-incision fascial release of leg compartments was performed under general anesthesia, as an emergency. Intravenous antibiotics (cefepime one gm twice a day) were started. Soon after, kidney derangement developed. Family members declined further treatment and took the patient home. This was at five weeks following the intra-articular injection. Intravenous antibiotics and regular dressing of leg were continued for next three weeks. During this period the patient continued to deteriorate. She developed pressure sores over her sacrum and trochanteric regions. It was in this state that she presented to our emergency.

The patient at presentation had haemoglobin of $6.2 \mathrm{~g} / \mathrm{dl}$ (normal range $=12-15 \mathrm{~g} / \mathrm{dl}$ ), total leukocyte count of $5.4 \times 10^{3} / \mathrm{mm}^{3}$ (normal range $=4-11 \times 10^{3} /$ $\mathrm{mm}^{3}$ ), platelets $0.96 \times 105 / \mathrm{mm}^{3}$ (normal range $=1.5$ $4.0 \times 10^{5} / \mathrm{mm}^{3}$ ), bleeding time of 5 minutes (normal range $=2-9$ minutes), prothrombin time of 30 seconds (control = 12 seconds). Serum creatinine was $2.1 \mathrm{mg} /$

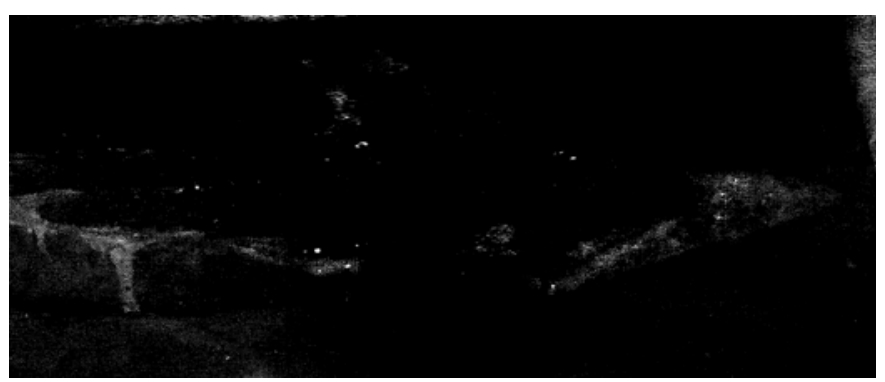

Fig 1: Patient with necrotising fasciitis of left lower limb following intra-articular left knee steroid injection. Presence of necrotic fascia present in surgical incisions and subcutaneous fat necrosis at the knee level is evident. dl (normal range $=0.6-1.1 \mathrm{mg} / \mathrm{dl}$ ), urea was $135 \mathrm{mg} /$ dl (normal range $=7-21 \mathrm{mg} / \mathrm{dl}$ ), sodium was 140.4 $\mathrm{mEq} / \mathrm{L}$ (normal range $=135-145 \mathrm{mEq} / \mathrm{L})$, potassium was $3.3 \mathrm{mEq} / \mathrm{L}$ (normal range $=3.5-5.0 \mathrm{mEq} / \mathrm{L}$ ) and blood sugar was $101 \mathrm{mg} / \mathrm{dl}$ (normal range $=80-110$ $\mathrm{mg} / \mathrm{dl}$ ). Blood cultures were sent. Attempted venous cannulation in cubital fossa resulted in a purulent ooze on puncturing the antebrachial fascia. A central venous catheter was inserted, intravenous cefepime one gm twice a day and intravenous teicoplannin $400 \mathrm{mg}$ once a day started. With support of inotropes her blood pressure was stabilised and five units of whole blood were transfused.

Patient's family was informed of a dismal prognosis, and the patient was shifted to operation theatre for emergency hip disarticulation in an attempt to debulk the septic focus. A racquet-shaped incision was made around the left hip. There was evidence of subcutaneous fat necrosis with intravascular coagulation seen in superficial veins (Fig.2). All the fascial septa were necrotic, with large purulent collections in thigh compartments, deep veins were thrombosed. The hip was disarticulated, and left lower limb removed (Fig. $3 \mathrm{a}, 3 \mathrm{~b}$ ). Muscles and superficial layers were closed over a corrugated rubber drain and stump dressing applied (Fig.4). The pressure sores over the trochanteric and sacral regions were debrided and dressed. The patient was shifted to intensive-care unit on ventilator support. Inotropes and antibiotics were continued.

Twenty four hours post surgery, the patient developed pneumonia and sudden hyperglycemia (blood glucose 358 milligrams per decilitre). She started bleeding from the surgical wound and vagina. Her blood pressure dropped to $40 / 30 \mathrm{~mm} \mathrm{Hg}$. Despite inotrope support, she succumbed to multi-system organ failure 36-hours after surgery. Her blood cultures subsequently grew Salmonella typhi sensitive to ciprofloxacin and ceftizoxime.

\section{DISCUSSION:}

Necrotising fasciitis (NF) is a progressive infectious process involving devitalisation of fascia and subcutaneous tissue, which becomes life threatening if not diagnosed and treated early. The infective process leading to fascial necrosis is the result of a mixed bacterial infection caused by aerobic and anaerobic bacteria. The pathogens most commonly involved are Group A Streptococci, Staphylococcus aureus, Peptostreptococcus and Enterobacteriaceae. ${ }^{12}$ Fasciitis necroticans is a rare disease and most of the patients developing it, have pre-existing conditions rendering them susceptible to infections. ${ }^{9}$ These contributory factors include diseases like systemic lupus erythematosus, multiple myeloma, chronic steroid use, chronic use of immunosuppressive medications. Severe pain disproportionate to local findings associated with systemic toxicity should raise the suspicion of necrotising fasciitis. ${ }^{13}$

Salmonella infection is usually associated with asymptomatic enteric carriage, gastroenteritis 


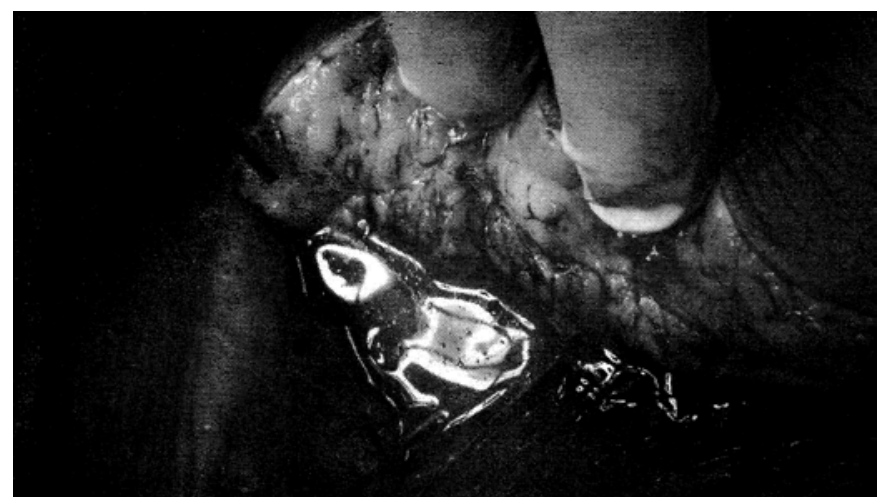

Fig 2: Operative wound showing presence of fat globules in the purulent exudates and evidence of intravascular coagulation noted in the thrombosed veins.
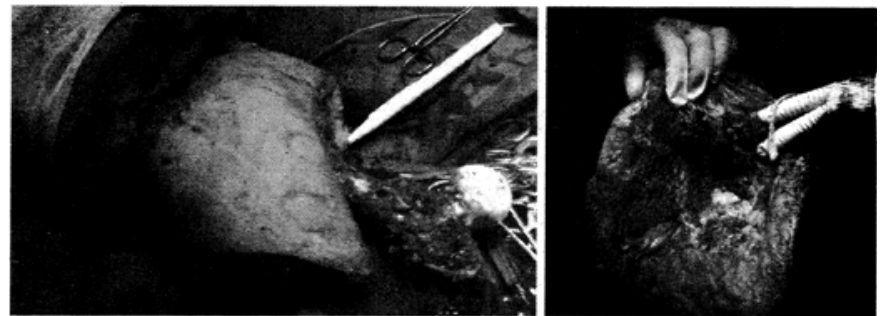

Figure $3 a, 3 b$ : Operative photographs showing disarticulation of left hip.

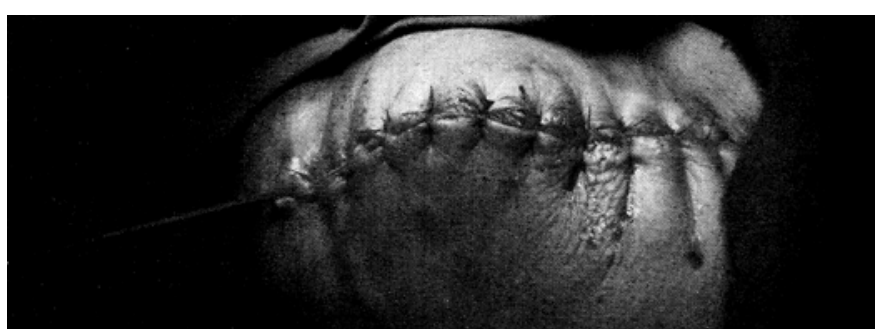

Fig 4: Amputation stump following disarticulation

and enteric fever. Extra-intestinal manifestations include endovascular infection, osteomyelitis, septic arthritis, and myonecrosis. ${ }^{10,14,15}$ Patients with underlying malignancy, diabetes, sickle cell disease, HIV infection and those on corticosteroids and immunosuppressive medications are at a greater risk of invasive salmonella infections. ${ }^{10}$ The synovium is a particular metastatic focus of Salmonella infection especially in immunocompromised patients. ${ }^{16}$ Complex lipopolysaccharides present in outer cell membrane of Salmonella behave as endotoxin. This freely circulating endotoxin activates both coagulation and fibrinolytic systems. DIC is produced during endotoxemia by combination of vasoconstriction, platelet damage, vascular endothelial damage and inhibition of local fibrinolysis. ${ }^{17}$

\section{CONCLUSION:}

Acute flare up of arthritis in cases of osteoarthritis knee can be managed conservatively with oral NSAIDs for short periods, and occasionally intra-articular steroid injections are administered. The steroid injection should be done taking all due anti-septic precautions. The older age-group of immunocompromised patients presenting with a tender knee may have underlying infection, which may flare up on injection of steroid. Such cases should be screened and investigated thoroughly before planning an injection treatment. If an unsuspected infection flares up, it should be diagnosed early and treated appropriately. Salmonella typhi is an uncommon cause of septic arthritis and necrotising fasciitis. Early joint aspiration and blood cultures may help detect the causative organism and control the infective process. Salmonella infection usually responds to third generation cephalosporin. DIC represents the end-point of endotoxemia. Gram negative sepsis should not be allowed to progress to this irreversible stage.

\section{REFERENCES:}

1. Birkinshaw R, O’ Donnel J, Sammy I. Necrotizing fasciitis as a complication of steroid injection. J Accid Emerg Med. 1997;14:52-4.

2. Regev A, Weinberger M, Fishman M, Samra Z, Pitlik SD. Necrotizing fasciitis caused by Staphilococcus Aureus. Eur J Clin Microbiol Infect Dis. 1998;17:101-3.

3. Hofmeister E, Engelhardt S. Necrotizing fasciitis as a complication of injection into greater trochanteric bursa. Am J Orthop. 2001;30:426-7.

4. Unglaub F, Guehring T, Fuchs PC, Perez-Bouza A, Groger A, Pallua N. Necrotizing fasciitis following therapeutic injection in a shoulder pain. Orthopade. 2005;34:250-2.

5. Fanfarillo F, Pace F, Maida R, Pignata D, Cergua G. Necrotizing fasciitis following intra-articular steroid injection: Case report and review of the literature. Geriatr Gerontol Int. 2012;12(2):353-5.

6. Rosser A, Swallow G, Swann RA, Chapman C. Salmonella enteritidis necrotising fasciitis in a multiple myeloma patient receiving bortezomib. Int J Haematol. 2010;91(1):149-51.

7. Luo CW, Liu CJ. Neck abscess and necrotizing fasciitis caused by Salmonella infection: a report of 2 cases. JOral Maxillofac Surg. 2007;65(5):1032-4.

8. Khawcharoenporn T, Apisarnthanarak A, Kiratisin P, Mundy LM. Salmonella group $C$ necrotizing fasciitis: a case report and review of the literature. Diagn Microbiol Infect Dis. 2006;54(4):319-22.

9. Andriessen MJ, Kotsopoulos AM, Bloemers FW, Strack van Schijndel RJ, Girbes AR. Necrotizing fasciitis caused by Salmonella enteritidis. Scand J Infect Dis. 2006;38(1112):1106-7.

10. Suwannaroj S, Mootsikapun P, Vipulakorn K, Nanagara R. Salmonella group D arthritis and necrotizing fasciitis in a patient with rheumatoid arthritis and diabetes mellitus. J Clin Rheumatol. 2001;7(2):83-5.

11. Sanchez C, Capell S, Casanovas A, Admetlla M, Sitges-Serra A. Necrotizing fasciitis caused by Salmonella enteritidis. Scand J Infect Dis. 1984;16(3):321-2.

12. 12. Wong CH, Chang HC, Pasupathy S, Khin LW, Tan JL, Low CO. Necrotizing fasciitis: clinical presentation, microbiology, and determinants of mortality. J Bone Joint Surg Am. 2003;85A(8):1454-60.

13. Hasham S, Matieucci P, Stanley PR, Hart NB. Necrotising fasciitis. BMJ. 2005;330(7495):830-3.

14. Jorring S, Kolmos HJ, Klareskov B. Myonecrosis in the leg caused by Salmonella enteritidis. Scand J Infect Dis. 1994;26(5):619-21.

15. Santos-Juanes J, Lopez-Escobar M, Galache $C$ et al. Haemorrhagic cellulitis caused by Salmonella enteritidis. Scand J Infect Dis. 2005;37(4):309-10.

16. Chen JY, Luo SF, Wu YJ, Wang CM, Ho HH. Salmonella Septic Arthritis in Systemic Lupus Erythematosus and Other Systemic Diseases. Clin Rheumatol. 1998;17(4):282-7.

17. Glover SC, Smith CC, Porter IA. Fatal Salmonella septicaemia with disseminated intravascular coagulation and renal failure. J Med Microbiol. 1982;15(1):117-21. 